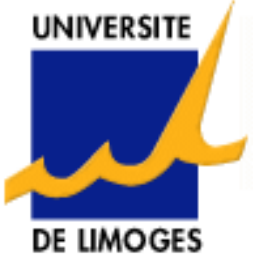

\author{
XLIM \\ UMR CNRS 6172

\section{Département \\ Mathématiques-Informatique}

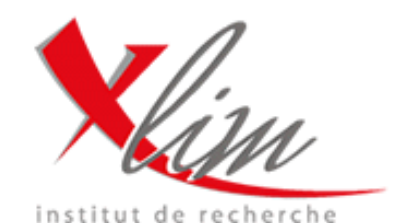

institut de recherche

On Directed Sets and their Suprema

M. Ait Mansour \& N. Popovici \& M. Théra

Rapport de recherche $\mathrm{n}^{\circ}$ 2006-03

Déposé le 4 janvier 2006 
Université de Limoges, 123 avenue Albert Thomas, 87060 Limoges Cedex

Tél. (33) 555457323 - Fax. (33) 555457322

http://www.xlim.fr

http://www.unilim.fr/laco 


\title{
ON DIRECTED SETS AND THEIR SUPREMA
}

\author{
M. AIT MANSOUR, N. POPOVICI, AND M. THÉRA
}

\begin{abstract}
The aim of this paper is to investigate real partially ordered linear topological spaces in which directed sets admit a supremum in their closure. In particular, we point out that this property is intimately related to the normality of the ordering cone and also to the Scott continuity of functionals belonging to the nonnegative polar of the ordering cone.
\end{abstract}

\section{Introduction And Preliminaries}

Throughout this paper $(E, \tau)$ will be a real linear topological space. For every $x \in E$, we denote by $\mathcal{V}(x)$ the family of all neighborhoods of $x$ (actually, $\tau$ being a linear topology, we have $\mathcal{V}(x)=x+\mathcal{V}\left(0_{E}\right)$, where $0_{E}$ stands for the zero-element of $E)$. As usual, $E^{\star}$ stands for the topological dual of $E$. By $w:=\sigma\left(E, E^{\star}\right)$ we mean the weak topology of $E$. For any subset $A$ of $E$, we denote by $\operatorname{cl} A$ (resp. $\operatorname{int} A$ ) the closure (resp. interior) of $A$ with respect to $\tau$, and by $w$-cl $A$ the closure of $A$ with respect to $w$.

In the sequel, the linear topological space $E$ will be partially ordered ordered by a convex cone $C$ assumed to be closed and proper (i.e., $\left\{0_{E}\right\} \neq C=\operatorname{cl} C \neq E$ ). As usual, the partial ordering (i.e., reflexive and transitive) relation is defined by:

$$
x \leqq_{C} y \Leftrightarrow y \in x+C
$$

Recall that $C$ is said to be pointed if $\ell(C):=C \cap(-C)=\left\{0_{E}\right\}$. Obviously, $C$ is pointed if and only if relation $\leqq_{C}$ is antisymmetric.

The polarity associated to $C$ (cf. [9]) is the mapping [·] $C: 2^{E} \rightarrow 2^{E}$, which assigns to each subset $A$ of $E$ the (closed convex) set

$$
[A]_{C}:=\bigcap_{x \in A}(x+C)=\left\{y \in E \mid \forall x \in A, x \leqq_{C} y\right\}
$$

2000 Mathematics Subject Classification. Primary 54F05; Secondary 06B30.

Key words and phrases. Directed and downward sets; Scott topologies; Normal cones; Daniell property.

Research of Mohamed Ait Mansour was supported by LACO (Laboratoire d'Arithmétique, Calcul Formel et Optimisation), UMR-CNRS 6090, University of Limoges and Agence Universitaire de la Francophonie. 
of all upper bounds of $A$. Of course, the set of lower bounds of $A$ can be represented by means of the polarity associated to $-C$, as

$$
[A]_{-C}:=\bigcap_{x \in A}(x-C)=\left\{z \in E \mid \forall x \in A, z \leqq_{C} x\right\} .
$$

The following preliminary result summarizes some basic properties of polarity mappings, which will be useful in the sequel. For a detailed study of polarities from the vector optimization point of view, we refer the reader to [9].

Lemma 1.1. The following properties hold:

(i) $[\emptyset]_{C}=E$ and $[E]_{C}=\emptyset$;

(ii) $[-C]_{C}=C$;

(iii) $\forall A \subset B \subset E,[A]_{C} \supset[B]_{C}$;

(iv) $\forall A \subset E, A \subset\left[[A]_{C}\right]_{-C}$.

As usual in vector optimization (see for instance [14]), the set of ideal maximal points of any subset $A$ of $E$ is defined as

$$
\operatorname{IMax} A:=A \cap[A]_{C},
$$

and the set of Pareto maximal points of $A$ is given by

$$
\begin{aligned}
\operatorname{Max} A & :=\{x \in A \mid A \cap(x+C) \subset x-\ell(C)\} \\
& =\left\{x \in A \mid \forall y \in A, x \leqq_{C} y \Rightarrow y \leqq_{C} x\right\}
\end{aligned}
$$

Analogously, by definition, the set of ideal minimal points of $A$ is

$$
\operatorname{IMin} A:=A \cap[A]_{-C},
$$

and the set of Pareto minimal points of $A$ is

$$
\begin{aligned}
\operatorname{Min} A & :=\{x \in A \mid A \cap(x-C) \subset x+\ell(C)\} \\
& =\left\{x \in A \mid \forall y \in A, y \leqq_{C} x \Rightarrow x \leqq_{C} y\right\}
\end{aligned}
$$

Remark 1.2. Obviously, $\operatorname{IMax} A \subset \operatorname{Max} A$ and $\operatorname{IMin} A \subset \operatorname{Min} A$. In fact, it is known (see e.g. [14, Proposition 2.2.2]) that $\operatorname{IMax} A=\operatorname{Max} A$ whenever $\operatorname{IMax} A$ is nonempty. Analogously, if IMin $A$ is nonempty, then $\operatorname{IMin} A=\operatorname{Min} A$.

Proposition 1.3. For every subset $A$ of $E$ we have

$$
\begin{aligned}
\operatorname{IMin}[A]_{C} & =\ell(C)+\operatorname{IMin}[A]_{C} \\
\operatorname{IMax}[A]_{-C} & =\ell(C)+\operatorname{IMax}[A]_{-C}
\end{aligned}
$$


Proof. Since $0_{E} \in \ell(C)$, the inclusion $\operatorname{IMin}[A]_{C} \subset \ell(C)+\operatorname{IMin}[A]_{C}$ is obvious. In order to prove the converse inclusion, let $c \in \ell(C)$ and $x \in \operatorname{IMin}[A]_{C}$. We have to show that $c+x \in[A]_{C}$ and $c+x \leqq_{C} y$ for all $y \in[A]_{C}$. Indeed, on one hand, $c+x \in$ $c+\operatorname{IMin}[A]_{C} \subset c+[A]_{C}=c+\bigcap_{a \in A}(a+C)=\bigcap_{a \in A}(a+c+C) \subset \bigcap_{a \in A}(a+C)=[A]_{C}$. On the other hand, since $x \in \operatorname{IMin}[A]_{C}$ and $c \leqq_{C} 0_{E}$, it follows that $c+x \leqq_{C} x \leqq_{C} y$ for all $y \in[A]_{C}$. Hence, the first desired equality, $\operatorname{IMin}[A]_{C}=\ell(C)+\operatorname{IMin}[A]_{C}$, holds. The second claimed equality may be proved by a similar argument.

Remark 1.4. If $C$ is pointed then, for any $A \subset E$, each of the sets IMax $A$ and IMin $A$ is either empty or it reduces to a singleton.

We say that a subset $A$ of $E$ admits a supremum (resp. infimum) if $\operatorname{IMin}[A]_{C}$ (resp. IMax $[A]_{-C}$ ) has cardinality one; in this case, the single element of $\operatorname{IMin}[A]_{C}$ (resp. IMax $[A]_{-C}$ ), the so-called supremum (resp. infimum) of $A$, will be denoted by $\sup A(\operatorname{resp} . \inf A)$.

Remark 1.5. By Proposition 1.3, we can easily conclude that if at least one subset $A$ of $E$ admits a supremum or an infimum, then $C$ is pointed.

A subset $A$ of $E$ is said to be upper bounded (resp. lower bounded) if $[A]_{C} \neq \emptyset$ (resp. $[A]_{-C} \neq \emptyset$ ); it is called order-bounded if it is both upper bounded and lower bounded. As usual (see e.g. [12]), $A$ is said to be bounded (with respect to $\tau$ ) if it is absorbed by every neighborhood of the origin, i.e., for every $V \in \mathcal{V}\left(0_{E}\right)$, there exists $t \in] 0,+\infty[$ such that $A \subset t V$. As shown below, the relationship between orderboundedness and usual (topological) boundedness may be established in terms of nonemptiness of the interior and normality of the ordering cone.

Lemma 1.6. If $C$ has nonempty interior, then every bounded subset of $E$ is orderbounded.

Proof. Let $A$ be a bounded subset of $E$. Pick an arbitrary $e \in \operatorname{int} C$. Then $e-C \in$ $\mathcal{V}\left(0_{E}\right)$ and $-e+C \in \mathcal{V}\left(0_{E}\right)$. Since $A$ is bounded, there exist $s, t>0$ such that $A \subset s(e-C)$ and $A \subset t(-e+C)$, i.e., $A \subset(-t e+C) \cap(s e-C)$, which shows that $A$ is order-bounded.

Recall (see e.g. [15] or [11]) that $C$ is called normal if $0_{E}$ admits a neighborhood basis consisting of full sets, i.e., for every $V \in \mathcal{V}\left(0_{E}\right)$ there exists $U \in \mathcal{V}\left(0_{E}\right)$ such that $U=(U+C) \cap(U-C) \subset V$.

Remark 1.7. If $C$ is normal, then every order-bounded subset of $E$ is bounded (see e.g. [15, Proposition 2.1.4]). 
Remark 1.8. If $E$ is Hausdorff and $C$ is normal, then $C$ is pointed; the converse is also true if, in addition, the Hausdorff space $E$ is assumed to be finite-dimensional (see for instance [11, Corollaries 2.1.23 and 2.2.11]).

By a vector lattice we mean a partially ordered vector space $\left(E, \leqq_{C}\right)$ (see for instance [15]) such that each pair of points $a$ and $b$ in $E$, admits a maximum and a minimum in the order induced by $C$. According to Remark 1.5 , if $\left(E, \leqq_{C}\right)$ is a lattice, $C$ is well understood to be pointed. We need to recall some more definitions needed for this study. A lattice $\left(E, \leqq_{C}\right)$ is said to be conditionally complete (see e.g. [3]) if every non-empty subset of $E$ with an upper bound admits a supremum (or, equivalently, if every non-empty subset of $E$ with a lower bound admits an infimum).

A subset $A$ of $E$ is called directed if for all $x, y \in A$, there exists $z \in A$ such that $x \leqq_{C} z$ and $y \leqq_{C} z$. Obviously, the empty set is directed; the space $E$ itself is directed if and only if $E=C-C$, i.e., $C$ is reproducing (see e.g. [13]). Note that $C$ is reproducing whenever $\left(E, \leqq_{C}\right)$ is a lattice or $\operatorname{int} C$ is nonempty, these two conditions being independent even in Banach spaces (see e.g. [4]).

The following class of sets will play a central role in the sequel:

$$
\mathcal{D}(E):=\left\{A \subset E \mid A \text { is directed and } \operatorname{IMin}[A]_{C} \neq \emptyset\right\} .
$$

Since the ordering cone $C$ is assumed to be proper, it is understood that $\emptyset \notin \mathcal{D}(E)$ and $E \notin \mathcal{D}(E)$ (even if $C$ is reproducing). Note also that, in view of Remark 1.4, if $C$ is pointed then we have

$$
\mathcal{D}(E)=\{A \subset E \mid A \text { is directed and admits a supremum }\} .
$$

In particular, if $\left(E, \leqq_{C}\right)$ is a conditionally complete lattice, then

$$
\mathcal{D}(E)=\{A \subset E \mid A \text { is nonempty, directed and upper bounded }\} \text {. }
$$

The principal aim of this paper is to investigate partially ordered linear topological spaces in which the following property holds:

$$
\operatorname{IMin}[A]_{C} \cap \operatorname{cl} A \neq \emptyset \quad \text { for all } \quad A \in \mathcal{D}(E) \text {. }
$$

The following example shows that Property (1) may fail even in Euclidean spaces, if they are ordered by certain closed convex proper cones.

Example 1.9. Let $E:=\mathbb{R}^{2}$ be the 2-dimensional Euclidean space, partially ordered by the (non pointed) closed convex cone $C:=\mathbb{R}_{+} \times \mathbb{R}$. Consider the set

$$
A:=\{(-1 / n, n) \mid n \in \mathbb{N}\} .
$$


It is easily seen that $A \in \mathcal{D}(E)$. However, since $[A]_{C}=\left[0,+\infty\left[\times \mathbb{R}, \operatorname{IMin}[A]_{C}=\right.\right.$ $\{0\} \times \mathbb{R}$, and $\operatorname{cl} A=A$, we have $\operatorname{IMin}[A]_{C} \cap \operatorname{cl} A=\emptyset$.

Remark 1.10. When $C$ is pointed, Property (1) becomes:

$$
\sup A \in \operatorname{cl} A \quad \text { for all } \quad A \in \mathcal{D}(E) .
$$

In particular, if $\left(E, \leqq_{C}\right)$ is a conditionally complete lattice, Property (2) means that $\sup A \in \operatorname{cl} A$ for every nonempty directed upper bounded subset $A$ of $E$.

On one hand, our study is motivated by a recent work [1], where Property (2) was crucial in establishing semi-continuous regularizations of vector-valued maps. Actually, for adequate level sets of a given vector-valued maps, Property (2) is shown to be intimately related to its semicontinuity. On the other hand, as we shall prove in this article, Property (2) characterizes those conditionally complete lattices whose nonnegative linear functionals are Scott continuous.

The paper is organized as follows: In Section 2 we investigate the relationship between polarities and topological closures of upward sets. In particular, we point out that condition IMin $[A]_{C} \cap \operatorname{cl} A \neq \emptyset$ in (1) means that $\operatorname{cl} A$ has at least one ideal maximal point. Then, we present our main results in Section 3. On one hand, we give a characterization of Property (2) in conditionally complete lattices, in terms of Scott continuity of nonnegative linear functionals. On the other hand, we show that Property (2) holds in reflexive Banach spaces, partially ordered by a normal cone with nonempty interior, and also in Hausdorff linear topological spaces, ordered by a lattice normal Daniell cone, under the hypothesis that any point has a countable basis of neighborhoods. Section 4 contains some concluding remarks and an open question.

\section{Polarities And Closures of DOWnWard Sets}

We begin this section by pointing out some links between polarities and topological closures.

Proposition 2.1. The following assertions hold for any subsets $A, B$ of $E$ :

(i) If $A \subset \operatorname{cl} B$ then $[\mathrm{cl} A]_{C} \supset[B]_{C}$;

(ii) If $\mathrm{cl} A=\mathrm{cl} B$ then $[A]_{C}=[B]_{C}$;

(iii) $[A]_{C}=[\operatorname{cl} A]_{C}$.

Proof. (i) Let $A, B \subset E$ be such that $A \subset \operatorname{cl} B$. Suppose to the contrary that there exists a point $\widetilde{b} \in[B]_{C} \backslash[\mathrm{cl} A]_{C}$. Then, on one hand, we have

$$
\widetilde{b} \in \bigcap_{b \in B}(b+C) .
$$


On the other hand, there exists $\widetilde{a} \in \operatorname{cl} A$ such that $\widetilde{b} \notin \widetilde{a}+C$. The cone $C$ being closed, the set $\widetilde{a}+C$ is closed too, hence there exists a neighborhood $V \in \mathcal{V}(\widetilde{b})$ with $V \cap(\widetilde{a}+C)=\emptyset$. Since $A \subset \operatorname{cl} B$ we have $\widetilde{a} \in \operatorname{cl} A \subset \operatorname{cl} B$ hence $\widetilde{a} \in \operatorname{cl} B$. By considering $\widetilde{a}+\widetilde{b}-V \in \mathcal{V}(\widetilde{a})$ it follows that $B \cap(\widetilde{a}+\widetilde{b}-V) \neq \emptyset$. Let $b_{0} \in B \cap(\widetilde{a}+\widetilde{b}-V)$. Then, by taking into account (3), we infer $b_{0} \in \widetilde{a}+b_{0}+C-V$, i.e., $\widetilde{a} \in V-C$. Let $v_{0} \in V$ and $c_{0} \in C$ be such that $\widetilde{a}=v_{0}-c_{0}$. Then $v_{0}=\widetilde{a}+c_{0} \in \widetilde{a}+C$, which yields $V \cap(\widetilde{a}+C) \neq \emptyset$, contradicting the choice of $V$.

(ii) Since $\operatorname{cl} A=\operatorname{cl} B$, we have $A \subset \operatorname{cl} B$ and $B \subset \operatorname{cl} A$. By (i) it follows that $[\mathrm{cl} A]_{C} \supset[B]_{C}$ and $[\mathrm{cl} B]_{C} \supset[A]_{C}$. Taking into account that $[\mathrm{cl} A]_{C} \subset[A]_{C}$ and $[\mathrm{cl} B]_{C} \subset[B]_{C}$, as shown by Lemma 1.1, we infer that $[A]_{C}=[B]_{C}$.

(iii) The conclusion follows by (ii) letting $B:=\operatorname{cl} A$.

A subset $A$ of $E$ is called downward (resp. upward) if $A=A-C$ (resp. $A=A+C$ ). Also known as sets possessing the free disposal property in the sense of Debreu [8], these sets play an important role in many fields of pure and applied mathematics (see e.g. [2]-[5] or [16] and references therein). In particular, in vector optimization, the study of Pareto maximal (resp. minimal) points of any set $A$ may be reduced to that of a downward (resp. upward) set. More precisely, we have $\operatorname{Max} A=\operatorname{Max}(A-C)$ and $\operatorname{Min} A=\operatorname{Min}(A+C)$. In concern with downward sets, we have the following results.

Proposition 2.2. For any subset $A$ of $E$ we have $[A-C]_{C}=[A]_{C}$.

Proof. By Lemma 1.1 we have:

$$
\begin{aligned}
{[A]_{C} } & =\bigcap_{a \in A}(a+C)=\bigcap_{a \in A}\left(a+[-C]_{C}\right) \\
& =\bigcap_{a \in A}\left(a+\bigcap_{x \in-C}(x+C)\right) \\
& =\bigcap_{a \in A}\left(\bigcap_{x \in-C}(a+x+C)\right) \\
& =\bigcap_{y \in A-C}(y+C)=[A-C]_{C} .
\end{aligned}
$$

Proposition 2.3. The closure of any downward set remains downward.

Proof. Let $A \subset E$. The inclusion $\operatorname{cl}(A-C) \subset \operatorname{cl}(A-C)-C$ obviously holds. In order to prove the converse inclusion, let $x \in \operatorname{cl}(A-C)-C$. Then $x=y_{0}-c_{0}$ for some $y_{0} \in \operatorname{cl}(A-C)$ and $c_{0} \in C$. Supposing to the contrary that $x \notin \operatorname{cl}(A-C)$, we can choose $V \in \mathcal{V}(x)$ such that $V \cap(A-C)=\emptyset$. Since $y_{0} \in \operatorname{cl}(A-C)$ we have $\left(V-x+y_{0}\right) \cap(A-C) \neq \emptyset$, hence there exist $a_{1} \in A$ and $c_{1} \in C$ with $a_{1}-c_{1} \in V-x+y_{0}$, i.e., $a_{1}-c_{1}+x-y_{0} \in V$. Recalling that $x=y_{0}-c_{0}$, it follows 
that $a_{1}-c_{1}-c_{0} \in V$, which shows that $a_{1}-c_{1}-c_{0} \in(A-C) \cap V$, contradicting the choice of $V$.

Corollary 2.4. For any subsets $A, B$ of $E$ such that $\mathrm{cl}(A-C)=\operatorname{cl}(B-C)$ we have $[A]_{C}=[B]_{C}$.

Proof. Directly follows by Proposition 2.1 and Proposition 2.2.

Remark 2.5. The converse of Corollary 2.4 is not true, as shown by the following example.

Example 2.6. Let $E:=\mathbb{R}^{2}$ be partially ordered by $C:=\mathbb{R}_{+}^{2}$, and let $A:=\{(0,0)\}$ and $B:=\{(-1,0) ;(0,-1)\}$. It is easily seen that $[A]_{C}=[B]_{C}=\mathbb{R}_{+}^{2}$, but $\operatorname{cl}(A-C)=$ $\left.\left.-\mathbb{R}_{+}^{2} \not \subset \operatorname{cl}(B-C)=-\mathbb{R}_{+}^{2} \backslash\right]-1,0\right]^{2}$.

The following result shows that condition (IMin $\left.[A]_{C}\right) \cap \operatorname{cl} A \neq \emptyset$ in (1) actually means that $\mathrm{cl} A$ has at least one ideal maximal point.

Theorem 2.7. For any subset $A$ of $E$ we have

$$
\left(\operatorname{IMin}[A]_{C}\right) \cap \operatorname{cl} A=\operatorname{IMax} \operatorname{cl} A=[A]_{C} \cap \operatorname{cl} A .
$$

Proof. Let us firstly note that, by Proposition 2.1, we have

$$
\operatorname{IMaxcl} A=[\operatorname{cl} A]_{C} \cap \operatorname{cl} A=[A]_{C} \cap \operatorname{cl} A \supset\left(\operatorname{IMin}[A]_{C}\right) \cap \operatorname{cl} A .
$$

On the other hand, by Lemma 1.1, we have $A \subset\left[[A]_{C}\right]_{-C}$. Taking into account that $\left[[A]_{C}\right]_{-C}$ is closed, we can deduce that $[A]_{C} \cap \operatorname{cl} A \subset \operatorname{cl} A \subset\left[[A]_{C}\right]_{-C}$. Hence

$$
[A]_{C} \cap \operatorname{cl} A \subset[A]_{C} \cap\left[[A]_{C}\right]_{-C}=\operatorname{IMin}[A]_{C},
$$

which shows that $[A]_{C} \cap \operatorname{cl} A \subset\left(\operatorname{IMin}[A]_{C}\right) \cap \operatorname{cl} A$. We conclude by combining (4) and (5).

Remark 2.8. Similar formulae to those presented in the previous result may be derived for $A-C$. Indeed, by Theorem 2.7 we get

$$
\operatorname{IMaxcl}(A-C)=[A-C]_{C} \cap \operatorname{cl}(A-C),
$$

which can be rewritten, thanks to Proposition 2.2, as

$$
\operatorname{IMaxcl}(A-C)=[A]_{C} \cap \operatorname{cl}(A-C) .
$$

Since $[A]_{C} \cap \operatorname{cl} A \subset[A]_{C} \cap \operatorname{cl}(A-C)$, by Theorem 2.7 and (6), we can deduce that

$$
\operatorname{IMax} \mathrm{cl} A \subset \operatorname{IMax} \mathrm{cl}(A-C) \text {. }
$$

The following result provides a sufficient condition in order to get equality in (7). 
Theorem 2.9. If $C$ is normal, then $\operatorname{IMax} \mathrm{cl}(A-C)=\operatorname{IMax} \operatorname{cl} A$ for every subset $A$ of $E$.

Proof. Let $\widetilde{x} \in \operatorname{IMax} \operatorname{cl}(A-C)$. Since $A-C$ is downward, by virtue of Proposition 2.3 we have $\operatorname{cl}(A-C) \subset \widetilde{x}-C \subset(\operatorname{cl}(A-C))-C=\operatorname{cl}(A-C)$, hence $\operatorname{cl}(A-C)=\widetilde{x}-C$. Consequently, we have $\operatorname{cl} A \subset \widetilde{x}-C$. Thus, in order to prove that $\widetilde{x} \in \operatorname{IMaxcl} A$, it suffices to prove that $\widetilde{x} \in \operatorname{cl} A$. Suppose to the contrary that $\widetilde{x} \notin \operatorname{cl} A$. Since $C$ is normal, we can find $V \in \mathcal{V}(\widetilde{x})$ such that $(V-C) \cap(V+C)=V$ and $V \cap A=\emptyset$. Recalling that $\widetilde{x} \in \operatorname{cl}(A-C)$, it follows that $V \cap(A-C) \neq \emptyset$. Let $x_{0} \in V \cap(A-C)$. Then $x_{0}=a_{0}-c_{0}$ for some $a_{0} \in A$ and $c_{0} \in C$. Since $\mathrm{cl} A \subset \widetilde{x}-C$, we deduce that $a_{0}=x_{0}+c_{0} \in x_{0}+C$ and $a_{0} \in A \subset \widetilde{x}-C$. Taking into account that both $x_{0}$ and $\widetilde{x}$ belong to $V$, we infer $a_{0} \in\left(x_{0}+C\right) \cap(\widetilde{x}-C) \subset(V+C) \cap(\widetilde{x}-C) \subset$ $(V+C) \cap(V-C)=V$. It follows that $a_{0} \in A \cap V$, contradicting the choice of $V$. Thus IMax $\mathrm{cl}(A-C) \subset \operatorname{IMax} \mathrm{cl} A$. The converse inclusion also holds by (7).

Remark 2.10. In view of Remark 1.8, the conclusion of Theorem 2.9 is true whenever $E$ is a finite-dimensional linear topological Hausdorff space and the closed convex cone $C$ is pointed. Note also that the hypothesis of normality is essential in Theorem 2.9 , as shown by the following example.

Example 2.11. Consider the set $A$ defined in Example 1.9. It is easily seen that $\operatorname{IMaxcl}(A-C)=\{0\} \times \mathbb{R} \not \subset \operatorname{IMaxcl} A=\operatorname{IMax} A=\emptyset$.

Corollary 2.12. Assume that $E$ is a linear topological Hausdorff space and $C$ is normal. Then, a subset $A$ of $E$ admits a supremum if and only if $A-C$ admits a supremum, $\sup A=\sup (A-C)$, and

$$
\sup A \in \operatorname{cl} A \Leftrightarrow \sup (A-C) \in \operatorname{cl}(A-C) .
$$

Proof. By Theorem 2.7 and Theorem 2.9 we can deduce that

$$
\begin{aligned}
\left(\operatorname{IMin}[A]_{C}\right) \cap \operatorname{cl} A & =\operatorname{IMaxcl} A \\
& =\operatorname{IMaxcl}(A-C) \\
& =\left(\operatorname{IMin}[A-C]_{C}\right) \cap \operatorname{cl}(A-C) .
\end{aligned}
$$

As mentioned in Remark 1.8, the cone $C$ is pointed, hence IMin $[A]_{C}=\{\sup A\}$ and $\operatorname{IMin}[A-C]_{C}=\{\sup (A-C)\}$. We infer that

$$
\{\sup A\} \cap \operatorname{cl} A=\{\sup (A-C)\} \cap \operatorname{cl}(A-C),
$$

completing the proof. 
The following result, which will be useful in the next section, concerns directed (downward) sets.

Proposition 2.13. If $A$ is a directed subset of $E$, then the following assertions hold:

(i) $A-C$ is directed;

(ii) $A-C$ is convex;

(iii) $\operatorname{Max} A=\operatorname{IMax} A$.

Proof. (i) Let $x, y \in A-C$. Then there exist $c, d \in C$ such that $x+c, y+d \in A$. Since $A$ is directed, there exists $z \in A \subset A-C$ such that $x \leqq_{C} x+c \leqq_{C} z$ and $y \leqq_{C} y+d \leqq_{C} z$. Hence $A-C$ is directed.

(ii) Let $x, y \in A-C$ and $t \in[0,1]$. Then there exist $a, a^{\prime} \in A$ and $c, c^{\prime} \in C$ such that $x=a-c$ and $y=a^{\prime}-c^{\prime}$. Since $A$ is directed, we can find a point $b \in A$ such that $a, a^{\prime} \in b-C$. As $C$ is convex, $b-C$ is convex, too. Thus, $(1-t) a+t a^{\prime} \in b-C$. Consequently, we have $(1-t) x+t y=(1-t) a+t a^{\prime}-\left[(1-t) c+t c^{\prime}\right] \in b-C-C=$ $b-C \subset A-C$. It follows that $A-C$ is convex.

(iii) The inclusion IMax $A \subset \operatorname{Max} A$ is obvious. To prove the converse one, let $x \in \operatorname{Max} A$. Consider an arbitrary $a \in A$. Since $A$ is directed, we can find some $b \in A$ such that $x \leqq_{C} b$ and $a \leqq_{C} b$. Given that $x \in \operatorname{Max} A$, it follows that $b \leqq_{C} x$, hence $a \leqq_{C} x$. Thus $x \in \operatorname{IMax} A$.

Remark 2.14. It is easily seen that a subset $A$ of $E$ is directed whenever $A-C$ is directed. However, the convexity of $A-C$ does not imply the convexity of $A$.

For concluding this section, we recall that the nonnegative polar cone (also known as dual cone) of $C$ is the convex cone defined by

$$
C^{+}:=\left\{\xi \in E^{\star} \mid \xi(x) \geq 0, \forall x \in C\right\},
$$

where $E^{\star}$ denotes the topological dual of $E$. It is well understood that a linear continuous functional, $\xi: E \rightarrow \mathbb{R}$, belongs to $C^{+}$if and only if $\xi(x) \leq \xi(y)$ for all $x, y \in E$ such that $x \leqq_{C} y$, i.e., $\xi$ is isotone with respect to $\leqq_{C}$ and the usual ordering $\leq$ from $\mathbb{R}$.

Corollary 2.15. For any subset $A$ of $E$, we have

$$
\operatorname{IMaxcl} A=\bigcap_{\xi \in C^{+}}\left(\operatorname{Argmin}_{x \in[A]_{C}} \xi(x) \cap \operatorname{Argmax}_{y \in \operatorname{cl} A} \xi(y)\right) .
$$

Proof. Firstly remark that

$$
\bigcap_{\xi \in C^{+}}\left(\operatorname{Argmin}_{x \in[A]_{C}} \xi(x) \cap \operatorname{Argmax}_{y \in \operatorname{cl} A} \xi(y)\right) \subset[A]_{C} \cap \operatorname{cl} A,
$$


since the null functional belongs to $C^{+}$. Now, let us prove that

$$
\operatorname{IMaxcl} A \subset \bigcap_{\xi \in C^{+}}\left(\operatorname{Argmin}_{x \in[A]_{C}} \xi(x) \cap \operatorname{Argmax}_{y \in \operatorname{cl} A} \xi(y)\right) .
$$

Let $x^{0} \in \operatorname{IMaxcl} A$ and let $\xi \in C^{+}$. Then $y \leqq_{C} x^{0}$, for all $y \in \operatorname{cl} A$ and $x^{0} \leqq_{C} x$, for all $x \in[\operatorname{cl} A]_{C}=[A]_{C}$. Hence $\xi(y) \leq \xi\left(x^{0}\right) \leq \xi(x)$, for all $y \in \operatorname{cl} A$ and $x \in[A]_{C}$. Since $x^{0} \in[\operatorname{cl} A]_{C} \cap \operatorname{cl} A=[A]_{C} \cap \operatorname{cl} A$, it follows that

$$
x^{0} \in \operatorname{Argmin}_{x \in[A]_{C}} \xi(x) \cap \operatorname{Argmax}_{y \in \operatorname{cl} A} \xi(y) .
$$

The conclusion follows from relations (8)-(9) and Theorem 2.7.

\section{MAin Results}

For all $\xi \in E^{\star}$ and $a \in \mathbb{R}$, let us denote

$$
H_{\xi, \alpha}:=\{x \in E \mid \xi(x)=\alpha\} \quad \text { and } \quad H_{\xi, \alpha}^{>}:=\{x \in E \mid \xi(x)>\alpha\} .
$$

Obviously, $H_{\xi, \alpha}$ is a closed hyperplane and $H_{\xi, \alpha}^{>}$is an open half-space whenever $\xi \in E^{\star} \backslash\left\{0^{\star}\right\}$, where $0^{\star}$ denotes the null functional. Note also that $H_{0^{\star}, \alpha}^{>}=\emptyset$ if $\alpha \geq 0$ and $H_{0^{\star}, \alpha}^{>}=E$ if $\alpha<0$.

Lemma 3.1. Let $\Phi: S \rightarrow 2^{E}$ be a set-valued mapping, defined on a nonempty set $S \subset 2^{E}$, which has the property that $\Phi(A) \cap \operatorname{cl} A \neq \emptyset$ for all $A \in S$. Then, for every $A \in S$ and every open $B \subset E$ with $\Phi(A) \subset B$ we have $A \cap B \neq \emptyset$.

Proof. Assume to the contrary that for some $A_{0} \in S$ and some open $B_{0} \subset E$ with $\Phi\left(A_{0}\right) \subset B_{0}$ we have $A_{0} \cap B_{0}=\emptyset$. Then we have $A_{0} \subset E \backslash B_{0}$, hence cl $A_{0} \subset E \backslash B_{0}$, which contradicts the fact that $\emptyset \neq \Phi\left(A_{0}\right) \cap \operatorname{cl} A_{0} \subset B_{0} \cap \operatorname{cl} A_{0}$.

Proposition 3.2. Assume that $\operatorname{IMin}[A]_{C} \cap \operatorname{cl} A \neq \emptyset$ for all $A \in \mathcal{D}(E)$. Then, for all $A \in \mathcal{D}(E)$ and $(\xi, \alpha) \in C^{+} \times \mathbb{R}$ with $\operatorname{IMin}[A]_{C} \subset H_{\xi, \alpha}^{>}$, we have $A \cap H_{\xi, \alpha}^{>} \neq \emptyset$.

Proof. Consider $S:=\mathcal{D}(E)$ and let us define the set-valued map $\Phi: S \rightarrow 2^{E}$ by $\Phi(A):=\operatorname{IMin}[A]_{C}$ for all $A \in S$. Then the conclusion directly follows from Lemma 3.1 , by using open sets $B$ of type $H_{\xi, \alpha}^{>}$.

As a straightforward consequence of Proposition 3.2 we obtain the following result.

Corollary 3.3. Assume that $C$ is pointed and $\sup A \in \operatorname{cl} A$ for all $A \in \mathcal{D}(E)$. Then $A \cap H_{\xi, \alpha}^{>} \neq \emptyset$, for all $A \in \mathcal{D}(E)$ and $(\xi, \alpha) \in C^{+} \times \mathbb{R}$ such that $\sup A \in H_{\xi, \alpha}^{>}$.

Theorem 3.4. Assume that $E$ is a locally convex Hausdorff space and that $C$ is normal. Let $A \in \mathcal{D}(E)$. Then, the following assertions are equivalent:

(i) $\sup A \in \operatorname{cl} A$; 
(ii) For all $\xi \in C^{+}$and $\alpha \in \mathbb{R}$ for which $\sup A \in H_{\xi, \alpha}^{>}$, we have $A \cap H_{\xi, \alpha}^{>} \neq \emptyset$.

Proof. In view of Remark 1.8, the cone $C$ is pointed, hence implication (i) $\Rightarrow$ (ii) follows by Corollary 3.3.

Conversely, assume that (ii) holds and suppose to the contrary that $\sup A \notin \operatorname{cl} A$. Consider the set $A_{0}:=A-C$. By Corollary 2.12, we infer that sup $A_{0} \notin \operatorname{cl} A_{0}$. On the other hand, by Proposition 2.13, $A_{0}$ is convex, hence $\mathrm{cl} A_{0}$ is convex, too. Thus, by virtue of a classical separation theorem (see e.g. [11, Theorem 2.2.8]), we can separate the point $\sup A_{0}$ from the nonempty closed convex set $\mathrm{cl} A_{0}$ by a closed hyperplane. More precisely, there exists $(\xi, \alpha) \in E^{\star} \times \mathbb{R}$ such that

$$
\sup \xi\left(\operatorname{cl} A_{0}\right) \leq \alpha<\xi\left(\sup A_{0}\right) .
$$

Let us prove that $\xi \in C^{+}$. To this end, let $c \in C$ be arbitrarily chosen. By (10) it follows that, for all $a \in A$ and $t \in] 0,+\infty$ [, we have $\xi(a-t c)<\xi\left(\sup A_{0}\right)$, i.e., $\frac{1}{t} \xi\left(a-\sup A_{0}\right)<\xi(c)$. Letting $t \rightarrow+\infty$, we infer that $\xi(c) \geq 0$. Thus $\xi \in C^{+}$. The second inequality in (10) shows that $\sup A_{0} \in H_{\xi, \alpha}^{>}$. By assumption (ii) it follows that $A_{0} \cap H_{\xi, \alpha}^{>} \neq \emptyset$, which contradicts the first inequality in (10).

Recall (see for instance [10] or [3]) that a set $U \subset E$ in a conditionally complete lattice $\left(E, \leqq_{C}\right)$ is said to be Scott open (open for the Scott topology of $E$ ) if $U$ is upward and for every $A \in \mathcal{D}(E)$ such that $\sup A \in U$ we have $A \cap U \neq \emptyset$. A function $f: E \rightarrow \mathbb{R}$ is called Scott continuous if it is continuous with respect to the Scott topologies of the conditionally complete lattices $\left(E, \leqq_{C}\right)$ and $(\mathbb{R}, \leq)$.

Remark 3.5. The Scott open sets in the conditionally complete lattice $(\mathbb{R}, \leq)$ are: $\emptyset$, $\mathbb{R}$, and all intervals of type $] \alpha,+\infty[$ with $\alpha \in \mathbb{R}$. Denoting by $\mathcal{U}$ the set of Scott open sets in $E$, it follows that for any function $f: E \rightarrow \mathbb{R}$ the following are equivalent (see Proposition 4.1, Theorem 4.2, and Theorem 4.3 in [3]):

$1^{\circ} f$ is Scott continuous.

$2^{\circ} f$ is lower semicontinuous for the Scott topology of $E$, i.e., for every $x \in E$ the following inequality holds:

$$
f(x) \leq \liminf _{y \rightarrow x} f(y):=\sup _{x \in U \in \mathcal{U}} \inf _{y \in U} f(y) \in \overline{\mathbb{R}} .
$$

$3^{\circ} f$ preserves upper bounded directed sups, that is,

$$
f(\sup D)=\sup f(D), \forall D \in \mathcal{D}(E) .
$$

Proposition 3.6. Assume that $E$ is a conditionally complete lattice. Then, for every $\xi \in C^{+}$, the following assertions are equivalent:

(i) $\xi$ is Scott continuous; 
(ii) For all $A \in \mathcal{D}(E)$ and $\alpha \in \mathbb{R}$ such that $\sup A \in H_{\xi, \alpha}^{>}$, we have $A \cap H_{\xi, \alpha}^{>} \neq \emptyset$.

Proof. (i) $\Rightarrow$ (ii). Consider some arbitrary $A \in \mathcal{D}(E)$ and $\alpha \in \mathbb{R}$ such that $\sup A \in$ $H_{\xi, \alpha}^{>}$. Taking into account that $H_{\xi, \alpha}^{>}=\xi^{-1}(] \alpha,+\infty[)$, we have $\sup A \in \xi^{-1}(] \alpha,+\infty[)$. Since $] \alpha,+\infty\left[\right.$ is Scott open in $\mathbb{R}$ (as shown by Remark 3.5), the set $\xi^{-1}(] \alpha,+\infty[$ ) is Scott open $E$, by assumption (i). Therefore, $A \cap \xi^{-1}(] \alpha,+\infty[) \neq \emptyset$, which means that $A \cap H_{\xi, \alpha}^{>} \neq \emptyset$.

(ii) $\Rightarrow$ (i). Let $T \subset \mathbb{R}$ be a Scott open set in $\mathbb{R}$. By Remark 3.5, there exists $\alpha \in \mathbb{R}$ such that $T=] \alpha,+\infty\left[\right.$. We have to prove that $\xi^{-1}(T)$ is Scott open in $E$. Obviously, $\xi^{-1}(T) \subset \xi^{-1}(T)+C$. To prove the converse inclusion, let $y \in \xi^{-1}(T)+C$. Then $y=x+c$ for some $x \in \xi^{-1}(T)$ and $c \in C$. Since $\xi \in C^{+}$, it follows that $\xi(y)=\xi(x+c)=\xi(x)+\xi(c) \in T+\mathbb{R}_{+}=T$, which shows that $y \in \xi^{-1}(T)$. Hence $\xi^{-1}(T)+C \subset \xi^{-1}(T)$. Thus the set $\xi^{-1}(T)$ is upward. Now, let $A \in \mathcal{D}(E)$ such that $\sup A \in \xi^{-1}(T)$. Then $\sup A \in H_{\xi, \alpha}^{>}$. By assumption (ii), we have $A \cap H_{\xi, \alpha}^{>} \neq \emptyset$, i.e., $A \cap \xi^{-1}(T) \neq \emptyset$. Consequently, $\xi$ is Scott continuous.

The following result gives a characterization of conditionally complete lattices with nonnegative Scott continuous linear functionals by means of Property (2).

Corollary 3.7. If $E$ is a conditionally complete lattice, then the following assertions are equivalent:

(i) $\sup A \in \operatorname{cl} A$ for all $A \in \mathcal{D}(E)$;

(ii) Each $\xi \in C^{+}$is Scott continuous.

Proof. Directly follows from Theorem 3.4 and Proposition 3.6.

A first class of partially ordered linear topological spaces satisfying Property (2) is exhibited in the next result.

Theorem 3.8. Assume that $E$ is a reflexive Banach space and $C$ is normal with nonempty interior. Then $\sup A \in \operatorname{cl} A$ for all $A \in \mathcal{D}(E)$.

Proof. Let $A \in \mathcal{D}(E)$. According to Corollary 2.12, it suffices to show that

$$
\sup (A-C) \in \operatorname{cl}(A-C) .
$$

Let us firstly prove that $\operatorname{cl}(A-C)$ is directed. To this end, let $x, y \in \operatorname{cl}(A-C)$. Then, there exist two sequences $\left(x_{n}\right)$ and $\left(y_{n}\right)$ in $A-C$ which converge to $x$ and $y$, respectively. Given that $A$ is directed, $A-C$ is also directed, as shown by Proposition 2.13. Thus, for every $n \in \mathbb{N}$ we can find a point $z_{n} \in A-C$ such that $x_{n} \leqq_{C} z_{n}$ and $y_{n} \leqq_{C} z_{n}$. The sequences $\left(x_{n}\right)$ and $\left(y_{n}\right)$ being bounded, the set $\bigcup_{n \in \mathbb{N}}\left\{x_{n}, y_{n}\right\}$ is 
also bounded, as well. Recalling that int $C \neq \emptyset$, the set $\bigcup_{n \in \mathbb{N}}\left\{x_{n}, y_{n}\right\}$ is also orderbounded, according to Remark 1.8. Thus, there exists $v \in E$ such that $v \leqq_{C} x_{n}$ and $v \leqq_{C} y_{n}$, hence $v \leqq_{C} z_{n}$, for all $n \in \mathbb{N}$. Obviously, the set $(v+C) \cap \operatorname{cl}(A-C)$ is lower bounded, a lower bound of it being $v$. On the other hand, by Proposition 2.1 and Proposition 2.2, we can deduce that $[A]_{C}=[\operatorname{cl}(A-C)]_{C}$, which shows that $\operatorname{cl}(A-C)$ is upper bounded, an upper bound of it being $\sup A$. Hence $(v+C) \cap \operatorname{cl}(A-C)$ is order-bounded. $C$ being normal, according to Remark 1.7, the set $(v+C) \cap \mathrm{cl}(A-C)$ is also bounded. Moreover, it is convex, as shown by Proposition 2.13. The Banach space $E$ being reflexive, $(v+C) \cap \operatorname{cl}(A-C)$ is weakly compact, as being a closed convex bounded set (see e.g. [7, Corollary III.19]). Since $z_{n} \in(v+C) \cap \operatorname{cl}(A-C)$ for all $n \in \mathbb{N}$, we infer the existence of a subsequence $\left(z_{n_{k}}\right)$ of $\left(z_{n}\right)$, which weakly converges to a point $z \in(v+C) \cap \operatorname{cl}(A-C)$. Since $z_{n_{k}}-x_{n_{k}}, z_{n_{k}}-y_{n_{k}} \in C$ for all $n \in \mathbb{N}$, by taking into account that sequences $\left(z_{n_{k}}-x_{n_{k}}\right),\left(z_{n_{k}}-y_{n_{k}}\right)$ weakly converge to $z-x$ and $z-y$, respectively, it follows that $z-x, z-y \in w$-cl $C$. On the other hand, since $C$ is closed convex, it is also weakly closed (see e.g. [7, Theorem III.7]). Hence $z-x, z-y \in w-\operatorname{cl} C=C$, i.e., $x \leqq_{C} z$ and $y \leqq_{C} z$. Thus $\operatorname{cl}(A-C)$ is directed.

Now, by applying Proposition 2.13 for $\mathrm{cl}(A-C)$ instead of $A$, we deduce that $\operatorname{Maxcl}(A-C)=\operatorname{IMaxcl}(A-C)$. Since $(v+C) \cap \operatorname{cl}(A-C)$ is nonempty weakly compact and $E$ is locally convex (indeed $E$ is a normed space), it follows by a classical argument in vector optimization (see e.g. [14, Theorem 2.3.3 and Lemma 2.3.5] that $\operatorname{Maxcl}(A-C)$ is nonempty. Hence, $\operatorname{IMaxcl}(A-C)$ is nonempty, as well. Finally, by Theorem 2.7, we infer that $\sup (A-C) \in \operatorname{cl}(A-C)$.

Example 3.9. Let $E$ be a real reflexive Banach space (for instance: the sequence space $l^{p}:=\left\{\left.\left(x_{i}\right)_{i \in \mathbb{N}} \in \mathbb{R}^{\mathbb{N}}\left|\sum_{i=1}^{\infty}\right| x_{i}\right|^{p}<+\infty\right\}$ with $\left.p \in\right] 1,+\infty[$, endowed with the norm $\|x\|:=\left(\sum_{i=1}^{\infty}\left|x_{i}\right|^{p}\right)^{1 / p}, \forall x=\left(x_{i}\right)_{i \in \mathbb{N}} \in l^{p}$; the space $L^{p}[a, b]$ with $\left.p \in\right] 1,+\infty[$ of equivalence classes of $p$-th power Lebesgue integrable functions on a compact interval $[a, b]$, endowed with the norm $\left.\|x\|:=\left(\int_{a}^{b}|x(t)|^{p} \mathrm{~d} x\right)^{1 / p}, \forall x \in \hat{x} \in L^{p}[a, b]\right)$ and let $E^{\star}$ be its topological dual. For every $\left.\varepsilon \in\right] 0,1\left[\right.$ and $x^{\star} \in E^{\star} \backslash\left\{0^{\star}\right\}$, the set

$$
C:=\left\{x \in E \mid x^{\star}(x) \geq \varepsilon\left\|x^{\star}\right\| \cdot\|x\|\right\}
$$

is known to be a closed convex normal cone with nonempty interior with respect to the norm topology (see [11], Example 1.1.3, pp. 2-3).

Remark that both natural ordering cones $l_{+}^{p}:=\left\{\left(x_{i}\right)_{i \in \mathbb{N}} \in l^{p} \mid x_{i} \geq 0, \forall i \in \mathbb{N}\right\}$ and $L_{+}^{p}[a, b]:=\left\{\hat{x} \in L^{p}[a, b] \mid \exists x \in \hat{x}: x(t) \geq 0\right.$ almost everywhere on $\left.[a, b]\right\}$ have empty interior with respect to the norm topology in $l^{p}$ and $L^{p}[a, b]$, respectively. 
Corollary 3.10. If $E$ is a finite-dimensional normed space and $C$ is pointed with nonempty interior, then $\sup A \in \operatorname{cl} A$ for all $A \in \mathcal{D}(E)$.

Proof. Recalling that any finite-dimensional normed space is reflexive, hence complete (see e.g. [12]), and taking into account that the ordering cone $C$ is normal (according to Remark 1.8), the conclusion directly follows by Theorem 3.8.

We end this section by showing that Property (2) is still true besides the reflexivity framework, if we impose some other assumptions on the ordering cone. Recall (see e.g. [11]) that a net $\left(x_{i}\right)_{i \in I}$ in $E$ (where $(I, \preceq)$ is understood to be a directed set) is called increasing (with respect to $C$ ) if $x_{i} \leqq_{C} x_{j}$ for all $i, j \in I$ with $i \preceq j$. The ordering cone $C$ is said to be Daniell if every upper bounded increasing net $\left(x_{i}\right)_{i \in I}$ in $E$ admits a supremum, $\sup \left\{x_{i} \mid i \in I\right\} \in E$, and converges to it. Note that every Daniell cone is pointed if $E$ is Hausdorff (for some other properties of Daniell cones we refer the reader to [6] and [11]).

A second class of partially ordered linear topological spaces satisfying Property (2) is pointed out by our last result:

Theorem 3.11. Assume that $E$ is a Hausdorff linear topological space such that $0_{E}$ has a countable basis of neighborhoods, $C$ is normal and Daniell, and $\left(E, \leqq_{C}\right)$ is a lattice. Then, $\sup A \in \mathrm{cl} A$ for all $A \in \mathcal{D}(E)$.

Proof. Let $A \in \mathcal{D}(E)$. The cone $C$ being normal, it suffices to show that

$$
\sup (A-C) \in \operatorname{cl}(A-C),
$$

as mentioned in the proof of Theorem 3.8.

Let us firstly show that $\operatorname{cl}(A-C)$ is directed. Let $x, y \in \operatorname{cl}(A-C)$. Since $0_{E}$ has a countable basis of neighborhoods, there exists two sequences $\left(x_{n}\right)$ and $\left(y_{n}\right)$ in $A-C$ converging to $x$ and $y$, respectively. The set $A$ being directed, by Proposition 2.13 it follows that $A-C$ is directed. Thus, we can find a sequence $\left(z_{n}\right)$ in $A-C$ such that $x_{n} \leq_{C} z_{n}$ and $y_{n} \leq_{C} z_{n}$ for all $n \in \mathbb{N}$. On the other hand, since $A-C$ is directed, for each $n \in \mathbb{N}$ there exists an upper bound $u_{n} \in A-C$ of the finite set $\left\{z_{1}, z_{2}, \ldots, z_{n}\right\}$. Recalling that $\left(E, \leqq_{C}\right)$ is a lattice, we can define, for every $n \in \mathbb{N}, v_{n}:=\sup \left\{z_{1}, \ldots, z_{n}\right\} \in E$. Clearly, $v_{n} \in u_{n}-C \in A-C-C=A-C$, hence $v_{n} \in A-C$, for all $n \in \mathbb{N}$. Note that, by construction, the sequence $\left(v_{n}\right)$ is increasing, i.e., $v_{n} \leqq_{C} v_{n+1}$ for all $n \in \mathbb{N}$ (indeed, $v_{n+1}$ is an upper bound of $\left\{z_{1}, \ldots, z_{n}\right\}$, hence $\left.\sup \left\{z_{1}, \ldots, z_{n}\right\} \leqq_{C} v_{n+1}\right)$. Note also that the sequence $\left(v_{n}\right)$ is upper bounded, since $A-C$ is upper bounded (indeed, recalling that $A \in \mathcal{D}(E)$, by Proposition 2.2 we have sup $A \in[A]_{C}=[A-C]_{C}$, hence $\left.[A-C]_{C} \neq \emptyset\right)$. The cone $C$ being Daniell, 
we infer that $\left(v_{n}\right)$ converges to $v:=\sup \left\{v_{n} \mid n \in \mathbb{N}\right\} \in \operatorname{cl}(A-C)$. Remark that $x_{n} \leqq_{C} z_{n} \leqq_{C} v_{n} \leqq_{C} v$ and $y_{n} \leqq_{C} z_{n} \leqq_{C} v_{n} \leqq_{C} v$ for all $n \in \mathbb{N}$. Taking into account that $C$ is closed and recalling that $\left(x_{n}\right)$ and $\left(y_{n}\right)$ converge to $x$ and $y$, respectively, it follows that $x \leqq_{C} v$ and $x \leqq_{C} v$. Hence $\mathrm{cl}(A-C)$ is directed.

By Proposition 2.13, we have $\operatorname{Maxcl}(A-C)=\operatorname{IMaxcl}(A-C)$. Since $C$ is Daniell and $\mathrm{cl}(A-C)$ is nonempty closed and upper bounded, it follows by a well known result in vector optimization (see e.g. [11, Proposition 3.2.17]) that $\operatorname{Maxcl}(A-C)$, i.e., IMax $\operatorname{cl}(A-C)$, is nonempty. We conclude that $\sup (A-C) \in \operatorname{cl}(A-C)$, by using Theorem 2.7 .

Remark 3.12. Under the hypotheses of Theorem 3.8 or Theorem 3.11 (even without normality of $C)$, the set $\mathrm{cl}(A-C)$ is directed whenever $A \in \mathcal{D}(E)$. Consequently, the closure of every downward set $A \in \mathcal{D}(E)$ is directed.

Example 3.13. Consider the space $E:=l^{1}=\left\{\left(x_{i}\right)_{i \in \mathbb{N}} \in \mathbb{R}^{\mathbb{N}}\left|\sum_{i=1}^{\infty}\right| x_{i} \mid<+\infty\right\}$, endowed with the norm $\|x\|:=\sum_{i=1}^{\infty}\left|x_{i}\right|, \forall x=\left(x_{i}\right)_{i \in \mathbb{N}} \in E$, and let $C$ be the natural ordering cone in $E$, defined by

$$
C:=l_{+}^{1}=\left\{x=\left(x_{i}\right)_{i \in \mathbb{N}} \mid x_{i} \geq 0, \forall i \in \mathbb{N}\right\} .
$$

Obviously, the normed space $E$ is a Hausdorff linear topological space and $0_{E}$ has a countable basis of neighborhoods. Moreover, $\left(E, \leqq_{C}\right)$ is a lattice (see [15], Example 1.6, pp.10-12) and the cone $C$ is both Daniell and normal for the norm topology (see [13], Example 1.48, p. 30). Note that the Banach space $l^{1}$ is not reflexive and the norm interior of the ordering cone $l_{+}^{1}$ is empty.

\section{Conclusions}

The aim of this paper was to study those partially ordered real linear topological vector spaces in which Property (2) holds. On one hand, Corollary 3.7 shows that conditionally complete lattices satisfying (2) actually have Scott continuous nonnegative linear functionals. On the other hand, Theorems 3.8 and 3.11 emphasize some important partially ordered linear topological spaces satisfying Property (2). An interesting open question is to know whether Property (2) characterizes (under suitable assumptions) certain properties of the ordering cones, such as normality or Daniell property. We also hope that our study will find some other interesting applications, since directed sets and Scott topologies are nowadays intensively studied in many mathematical and theoretical computer science papers.

Acknowledgments The authors thank two anonymous referees for their useful remarks, leading to an improvement of the presentation. 


\section{REFERENCES}

[1] M. Ait Mansour, A. Metrane and M. Théra. Lower semicontinuous regularization for vector valued mappings, Journal of Global Optimization, (special issue dedicated to A. Rubinov, 2005, to appear).

[2] M. Akian (1999). Densities of idempotent measures and large deviations, Trans. Amer. Math. Soc., 351, 4515-4543.

[3] A. Akian and I. Singer (2003). Topologies on lattice ordered groups, separations from closed downwards and conjugations of type Lau, Optimization, 52, 629-673.

[4] J. Benoist, J. M. Borwein and N. Popovici (2003). A Characterization of Quasiconvex VectorValued Functions. Proc. Amer. Math. Soc., 131, 1109-1113.

[5] J. Benoist and N. Popovici (2000). The structure of the efficient frontier of finite-dimensional completely-shaded sets. J. Math. Anal. Appl., 250, 98-117.

[6] J. M. Borwein (1982). Continuity and differentiability properties of convex operators. Proc. London Math. Soc., 44, 420-444.

[7] H. Brézis. Analyse fonctionnelle: Théorie et applications. Masson, Paris, 1983.

[8] G. Debreu. Theory of Value. John Wiley, New-York, 1959.

[9] S. Doleczki and C. Malivert (1988). Stability of efficient sets: continuity of mobile polarities, Nonlinear Anal., 12, 1461-1486.

[10] G. Gierz, K. H. Hoffmann, K. Keimel, J. D. Lawson, M. Mislove and D. S. Scott. A compendium of continuous lattices. Springer-Verlag, Berlin - New York, 1980.

[11] A. Göpfert, H. Riahi, C. Tammer and C. Zălinescu. Variational methods in partially ordered spaces. Springer-Verlag, New York, 2003.

[12] R. B. Holmes. Geometric functional analysis and its applications. Springer-Verlag, New York - Heidelberg, 1975.

[13] J. Jahn. Mathematical vector optimization in partially ordered linear spaces. Verlag Peter Lang, Frankfurt am Main, 1986.

[14] Dinh The Luc. Theory of vector optimization. Springer-Verlag, Berlin, 1989.

[15] A. L. Peressini. Ordered topological vector spaces. Harper and Row Publishers, New York, 1967.

[16] A. Rubinov. Abstract Convexity and Global Optimization, Kluwer Academic Publishers, Dordrecht, 2000.

Faculté des Sciences et Techniques, LACO - UMR 6090, 87060 Limoges cedex, FRANCE

E-mail address: mohamed.ait-mansour@unilim.fr

Babe s-Bolyai University, Faculty of Mathematics and Computer Science, 3400 Cluj-Napoca, Romania

E-mail address: popovici@math.ubbcluj.ro

Faculté des Sciences et Techniques, LACO-UMR 6090, 87060 Limoges Cedex, France

E-mail address: michel.thera@unilim.fr 